\title{
Mothers' Experiences of Kangaroo Mother Care During Hospitalization of Their Preterm Babies at an Academic Hospital in Johannesburg
}

\author{
Titus Kipchumba Tarus ${ }^{1,}$, , Adele Agatha Tjale ${ }^{2}$ \\ ${ }^{1}$ Nursing department, Moi Teaching and Referral Hospital, Eldoret, Kenya \\ ${ }^{2}$ Faculty of Health sciences, Department of Nursing education, University of the Witwatersrand, Johannesburg, South Africa
}

Email address:

kesigich@yahoo.com (T. K. Tarus), adele.tjale@wits.ac.za (A. A. Tjale)

\section{To cite this article:}

Titus Kipchumba Tarus, Adele Agatha Tjale. Mothers' Experiences of Kangaroo Mother Care During Hospitalization of Their Preterm Babies at an Academic Hospital in Johannesburg. American Journal of Nursing Science. Vol. 4, No. 4, 2015, pp. 200-206.

doi: 10.11648/j.ajns.20150404.18

\begin{abstract}
Kangaroo mother care (KMC) implies placing the newborn pre-term baby in intimate skin-to-skin contact with the mother's chest and abdomen coupled with frequent and preferably exclusive breast-feeding. This is similar to marsupial care-giving, where the premature baby is kept warm in the maternal pouch and close to the breasts for unlimited feeding. KMC has emerged as a non-conventional low cost method for newborn care that provides warmth, touch, and security to the newborn and is believed to confer significant survival benefit. The purpose of this study was to understand the mothers lived experience and perception towards 24-hour Kangaroo Mother Care (KMC) during hospitalization of their preterm babies. Phenomenological study methods were applied through in-depth unstructured interviews, on purposefully selected participants. Their lived experience was analyzed using Collaizzi's steps to phenomenological data analysis. Data analysis revealed three major themes: (a) "it is a bond between me and my child" (b) nurse-parent interaction, and (c) "it is tiring and exhaustive". The study found that all the mothers in spite of exhaustion, reported high level of satisfaction with KMC because it allowed them to be closer to their babies, hence giving them the opportunity to observe their growth and became fully involved in the care. The excellent nurse-mothers' interaction reinforced the KMC concept in care of preterm babies and was rated significant in the transition period of motherhood. The interaction enabled mothers to shift from passive observers to active participants in the care of the preterm babies. KMC was additionally noted to have provided a comforting and warm environment for the babies, thus enabling them to grow faster via weight gains and this in turn increased maternal satisfaction. Recommendation for further research preferably using quantitative methods to establish relationship between production of breast milk and the use of KMC is envisaged. The principle of 24-hour KMC should also be incorporated and emphasized in all levels of preterm baby care hospitals and educational training as this practice has been found to have numerous benefits both to the mothers and to their preterm babies.
\end{abstract}

Keywords: Preterm Babies, Kangaroo Mother Care, Hospitalized, Lived Experience

\section{Introduction}

Kangaroo mother care (KMC) is an innovative method used to promote closeness between a preterm baby and the mother. It involves placing a pre-term baby covered in an upright position between the mother's breasts for skin-to-skin contact. The name 'kangaroo' was obtained from the similarities it bears with marsupial care-giving, in which the baby marsupial born prematurely is guided into the maternal pouch where he is kept warm with easy access to the mother's breast for unlimited breastfeeding opportunities until maturation[1,2].
$\mathrm{KMC}$ is fronted as a low-cost strategy for pre-term baby care that provides warmth, believed to give significant survival to the pre-term baby, given their inability to control their body temperatures. It's often practiced and recommended in settings where there is shortage of staff and incubators, with unacceptable high mortality and infection rates in the neonatal unit, and burden of a large number of babies being abandoned by their mothers [3].

$\mathrm{KMC}$ had been introduced at the academic hospital as 
prompted by overcrowding noted from bed occupancy of more than $150 \%$ at the hospital's preterm baby unit. A prospective, randomized, controlled study in the CHB hospital had asserted benefits of KMC to be safe; because of the reduced exposure to infections, normal growth, frequent breastfeeding, and reduced hospital stay noted in the intervention group [4]. Owing to the reported immense benefits of KMC at the CHB hospital, we set off to find out the mothers' lived experience of the practice at the hospital.

Several comparative studies have been commissioned to establish effectiveness between KMC and conventional methods of care in both developed and developing countries, with most concluding that $\mathrm{KMC}$ offers additional advantages, regarding mother's empowerment and family bonding to the preterm babies [5] Physiologically, mother's breasts respond to baby's thermal needs. Mother's temperature rises when the baby's temperature is low [6]. In addition, other studies have indicated significant positive findings in weight gain, high survival rates, early initiation of breastfeeding, strong maternal-infant attachment, shorter hospital stay, and reduced parental stress in $\mathrm{KMC}$ as compared to conventional forms of care [7-9]. In support of less maternal stress during KMC, a study of 488 premature infants done in Bogota, Columbia found that KMC mothers not only showed a stronger bonding effect and a change in their perception of their child, but also a resilience effect and improved maternal satisfaction. KMC was also reported to play a role in reducing the incidence of severe illness and nosocomial infections and initiating early breastfeeding [9-12].

At the academic hospital where the study was carried out, the experiences and perceptions of mothers involved with the care have not been established and no published research in South Africa had been found in relation to mothers' lived experiences with KMC.

\section{Methods}

The study was a qualitative study design grounded on phenomenological, explorative, descriptive and contextual research approaches. Participants who were deemed knowledgeable about KMC were selected through purposive sampling. This involved participants who knew the most about the phenomenon and who were able to articulate and explain their nuances. In particular the participants were consenting mothers aged from 18years and above, who had their preterm babies admitted in the KMC ward for at least 7 days prior to the in-depth interviews. In addition the mothers were able to comprehend and speak English fluently as well as their admitted babies being free of illnesses or congenital abnormalities.

Data was collected through in-depth unstructured recorded interviews using audiotapes and supplemented with field notes. At the beginning of each interview, the researcher explained the purpose of the study to each prospective participant and sought their co-operation. The data was then transcribed verbatim on paper for discussion of the research.

Data analysis was conducted through Colaizzi's (1978) method of phenomenological analysis [13] whereby significant statements were extracted from each transcript and then categorized in a cluster of themes that are discussed as findings of the study.

Ethical approval for the study protocols was provided from various authorities including University of Witwatersrand's postgraduate committee, University of Witwatersrand's Human Research Ethics Committee, Provincial Research Committee, Department of health, Gauteng Province and the Chief Executive Officer of CHB Hospital.

\section{Results}

\subsection{Socio-Demographics of Participants}

Saturation of themes was achieved after interviewing 9 mothers whose characteristics are summarized in table 1 below.

Table 1. Profile of participants.

\begin{tabular}{lllllll}
\hline $\begin{array}{l}\text { Participants' } \\
\text { pseudonym }\end{array}$ & $\begin{array}{l}\text { Age } \\
(\mathbf{y r s})\end{array}$ & Race & $\begin{array}{l}\text { Duration in } \\
\text { KMC ward }\end{array}$ & $\begin{array}{l}\text { Number of alive } \\
\text { children }\end{array}$ & Marital status & $\begin{array}{l}\text { Baby's mode of } \\
\text { feeding }\end{array}$ \\
\hline Amikai & 28 & Black & 7 days & 2 & Married & Formula milk \\
Gloria & 38 & Black & 7 days & 5 & Married & Breastfeeding \\
Lissi & 26 & 'Coloured' & 10 days & 3 & Engaged, cohabiting & Breastfeeding \\
Maggie & 34 & Black & 13 days & 3 & Single, cohabiting & Breastfeeding \\
Maria & 18 & Black & 8 days & 1 & Single & Breastfeeding \\
Pearl & 19 & Black & 11 days & 1 & Single & Breastfeeding \\
Precious & 22 & Black & 7 days & 1 & Single & Breastfeeding \\
Rachael & 30 & 'Coloured' & 14 days & 3 & Single & Breastfeeding \\
Rebecca & 26 & Black & 13 days & 2 & Single, cohabiting & Breastfeeding \\
\hline
\end{tabular}

\subsection{Cluster of Themes}

The exhaustive description of mothers' experiences and perceptions of 24-hour KMC was at this stage written down in unambiguous statements. Similar themes were grouped together and synthesized into broader, yet more descriptive, themes that were common to all the transcripts. Three major themes emerged, each having two or more sub-themes (table 2) which were later reduced into a common narrative describing the mothers' experiences and perception of 24-hour KMC in the hospital setting. 
Table 2. KMC Themes and sub-themes.

\begin{tabular}{ll}
\hline Major themes & Sub-themes \\
\hline $\begin{array}{l}\text { It is a bond between my } \\
\text { child and myself }\end{array}$ & $\begin{array}{l}\text { I am able to play and talk with him/her } \\
\text { My baby is close to me during KMC sessions }\end{array}$ \\
$\begin{array}{l}\text { Iurse-mothers } \\
\text { I am able to give love to my child with KMC. } \\
\text { "Theraction }\end{array}$ & $\begin{array}{l}\text { Partnership in care } \\
\text { "It is tiring and }\end{array}$ \\
$\begin{array}{l}\text { "We don't sleep" } \\
\text { exhaustive" }\end{array}$ & "My back is sore" \\
\hline
\end{tabular}

\subsection{1. (Main Theme 1) It is a Bond Between My Child and} Me

All nine of the study participants described bonding as an ongoing affectionate process that takes place between the mother and her preterm baby during their close contact, talking, playing and breastfeeding. As a result of maternal-infant interactions, close contact, and commitment to love and care, attachment process progresses between the mother and her baby and endures over time as the mother comes to know, love, and accept her baby as explained by one participant .

"Kangaroo care is lovely because it is a bond between me and my child" (Rachael).

\section{(i). (Sub-Theme 1) I Play and Talk with $\mathrm{Him} / \mathrm{Her}$}

All the participants in this study described maternal-infant attachment as developing during interaction with their preterm babies. Talking and playing is termed in the literature search as maternal-infant interaction that takes place between the mother and her baby during the attachment process. These interactions involved talking, touching, gazing, smiling, and playing. It takes place even when the baby is asleep or breastfeeding.

Two participants said:

"When he is not asleep, I just talk with him, play with him" (Amikai).

"I sometimes just look at his face and smile. It makes me happy; most of the time I enjoy watching him sleeping" (Lissi).

These behaviors do not occur in a sequence but instead each individual behavior pattern triggers several others. For example, a baby's cry may prompt other forms of interactions listed above such as mother's touch, rocking and kissing to soothe the baby.

One participant explained:

"When my child cries I just play with him and talk with him" (Maggie)

At the CHB hospital preterm babies weighing less than 1200 grams are immediately separated from their mothers after birth and admitted to the NICU for specialized treatment. Mothers are then discharged home and only allowed to visit their babies at daybreak. For example, two participants expressed their joy while in the KMC ward:

"I am happy about KC[kangaroo care] because I am with my baby throughout but before [prior to KMC] I was with my baby only during the day; at night I wasn't sleeping - thinking about my baby all the time; I am now the one feeding, changing [the nappies], you know ... I am happy for that" (Maggie).

"KC is very nice... I am now with her for 24 hours" (Precious).

According to most of the participants in this study, fear hindered them from freely touching their babies while in the NICU. This could have had a negative impact on a normal attachment process that is expected to begin soon after birth.

"I couldn't touch the baby because he was very tiny, small. It took me four days to touch the baby and feel the baby; I told myself may be they have changed my baby; this is not mine" (Rebecca).

Some participants who were initially afraid to touch their babies were then able to interact with their babies freely during KMC. One mother explains:

"Before, I was so scared; I was so scared because she was so small; I couldn't even wash her; it is my first child ... so you understand why I was so scared; I kept looking at her; I had never touched her until here [KMC ward] and as time goes by, I became fine and accepted; actually when I see her now I see a big child; I am used to her now; I am no longer scared of anything" (Precious).

\section{(ii). (Sub-Theme2) My Baby is Close to Me}

Maternal-infant closeness was described by all participants to have had a link in bonding them with their babies because of being with them 24 hours a day. In addition, each participant perceived a close relationship between the baby's safety and closeness during the attachment process and breast milk production. Although most of the participants did not use the word 'safety', their descriptions of closeness linked KMC to the easy monitoring of their babies with regard to any potential complications.

"When providing $\mathrm{KC}$ to the child, it is easy to see her if in case any changes like color or anything happens to her. It is easy to see. Even breathing you can hear the heart beat when she is in your chest but in the incubator, you won't be able to notice that earlier. In the incubator she is not all that close like in my chest" (Precious).

A similar experience was shared by another participant who stated:

"You know sometimes 'nneh' one thing about kangaroo care is that the baby is with you but when she is in the bed you can't hear if something goes wrong with her" (Gloria).

Evidence of attachment during $\mathrm{KMC}$ is noted when a mother and her baby are temporarily separated. For example, one participant in this study who was temporarily separated from her baby when she was taken for specialized treatment experienced a feeling of loneliness within 12 hours even though she could deliver her usual maternal services such as cleaning, changing of nappies and feeding. She said:

"I feel lonely because I am used to having her close to me; kangaroo is a nice experience" (Rachael).

Similarly, another participant reported that her baby would feel uncomfortable even with a short separation.

"My child cries whenever I am not with her until I come back... that's when I leave her and go to the toilet" (Gloria). 
They argued that mothers' closeness to their babies during KMC enables them to become familiar with each other's cues. For instance, one participant in this study said:

"My baby gets to know me better because I am next to her. She gets used to the smell; in the incubator she is not all that close like in my chest" (Precious).

One participant who had twins initially did not have enough breast milk for her two babies but observed an improvement after starting to provide KMC.

"My milk wasn't enough to accommodate my two babies before but now is much better ...the milk is just coming out fine; it can accommodate the two of them" (Lissi).

\section{(iii). (Subtheme3)I Give Love to My Child}

Each participant had an experience that creates responsibilities and choices that benefit the baby describing this sub-theme. All participants in this study felt responsible for the love, growth and development of their babies. For example, some participants said:

"I just give love to my child wholly. Kangaroo care wants you, a real mother... because sometimes you feel so tired but if you are a mother, you can do it. You can't feel tired because you like it and you want your child to grow up but if you are not a real mother you can't do this [KMC]; premature baby needs love, strong care, you see" (Amikai).

"You must be happy, you must be friendly, you must talk [while providing $\mathrm{KMC}$ ] and if there is anything worrying you then you must call the sister(nurse) and say I have a problem like this and this and she can help you" (Maggie).

"If you are stressed it affects the baby; it affects the weight... especially when breastfeeding because of the bond... the child sense anything that goes on in your life" (Rachael).

"At first I didn't believe in this thing called KMC, I thought it was a waste of time but since I came here to KMC ward my baby has grown; it helps the baby to grow; I then started believing in this thing, it is amazing; it is working; I am happier my baby is growing because of KC" (Lissi).

"Kangaroo care is a good thing because it keeps the baby warm and make the baby grow faster; this kangaroo thing is still an incubator'(Rebecca)

\subsection{3. (Main Theme2) Nurse-Mothers Interaction}

The nurse-parent interaction was described by all participants as helpful in acquiring knowledge and getting into partnership to care of the baby. The partnership with the nurse was of great significant in the period of transition to motherhood and adjustment in the KMC ward. Some of the expectations noted in this study included mothers' desire to acquire information regarding their babies and $\mathrm{KMC}$ which was new to all, close supervision, and to be involved as partners in the care of their babies.

Mothers were satisfied with the nurse-parent interaction because of the role that nurses played in helping them during the transition period into motherhood and adjustment to KMC through their teaching, support, guidance and monitoring.

\section{(i). (Sub-Theme1) They (Nurses) Taught Us}

All participants needed information regarding their preterm babies and the care during their transitional period to motherhood. From each description, nurses played a crucial role in helping them to learn how to care for their babies during this period.

"They [Nurses] have taught us to look at the legs of the baby, the hands, finger tips ... you see and toe nails; we have also been taught what you can do when the baby reacts that way, ..." (Pearl).

Knowledge acquired during 24-hour KMC was highly appreciated by each participant because their preterm babies were prone to complications and such knowledge prepared them for any such crisis. In their explanations of what they had learnt, participants said:

"I know the color of my child is pink, so if I look at her and see a color like blue, I know that there is something wrong with my child; I also know how she breathes; if she can breathe slower or faster, I can tell" (Precious).

"What I have also learnt here is that when doing $\mathrm{KC}$, you have to be observant; it might happen that your baby changes color, you see... like in less oxygen" (Pearl).

"It is something new I have learnt. I never knew about $\mathrm{KC}$ and so they [nurses] taught me the first time I came here [KMC ward]; I want now to take my baby home... I know how to take care of him because I have the experience and I can do it for myself; I am not complaining now about KC... it is a nice experience... it is lovely" (Rachael).

"I got discouraged [when the weight of her baby dropped] and couldn't believe in it... but later I got encouragement when the nurse talked to me about $\mathrm{KC}$ and explained everything" (Lissi).

\section{(ii). (Sub-Theme2) Partnership in Care}

Participants expressed satisfaction during their babies' hospitalization for being treated by nurses as partners in the care of their babies. Partnership in care started while their babies were still in the NICU. Participants' involvement in baby care was expressed through statements like:

"When he was off medication, one sister came to me and asked me if I knew about KMC. I asked her many questions. What does it do? How does it help my baby... things like that (Pearl).

We were breastfeeding the babies and after she put her baby inside [her chest], she could not feel the baby breathing. Due to this she looked at him and the baby's mouth was bluish and the sister (nurse) helped her... the sister took the baby back to the ICU" (Rebecca).

"When I came here I felt at home; $\mathrm{KC}$ is so exciting" (Pearl).

Partnership in care enables mothers to shift from being passive observers to active participants. Further the positive nurse-mother relationship in KMC enabled mothers to freely express their feelings, ideas or disappointments whenever affected. One participant mentioned the following example:

"Whenever the sister hurts me I go to her and express my feelings and as soon as she apologizes it gets finished like that" 


\section{(Maggie).}

\subsection{3. (Main Theme3) It is Tiring and Exhaustive}

Participants described their experience in the KMC ward as including a consistent feeling of tiredness during 24-hour $\mathrm{KMC}$ as a result of inadequate sleep, high body temperatures, and the use of one position for sleep and rest, a common problem among postnatal mothers referred to as fatigue.

\section{(i). (Sub-theme1) We don't Sleep}

Inadequate sleep was reported due to interruptions to the normal sleep cycles. These disruptions were because of the routine three-hourly baby care provided by the mothers to their preterm babies. These included feeding and the changing of nappies. Besides this, the sleep according to some participants was not comfortable because their babies lay on their chests while they slept.

"You don't sleep normally, you are always awake, nurses are always here, they check on you, you change the nappies, feed the baby; you don't sleep nicely; besides that you don't sleep normally, you sleep with the baby; that is not normal, baby in the chest, that is not normal" (Rebecca).

"Oh ... I was so tired, I felt like crying; I felt like crying because my eyes were painful but my baby kept me strong because I love my child; I told myself that I have to be strong; I shouldn't allow my tiredness to overpower me" (Precious).

In summary of these individual experiences, all of the participants in this study initially found 24-hour KMC to be tiring.

\section{(ii). (Sub-Theme2) My Back Is Sore}

All participants in this KMC ward were expected to be in reclined or semi-recumbent positions whenever they wanted to sleep or rest as observed in figure 1. Each participant described this position as a precursor to backache and fatigue during KMC. Backache was a common problem among all nine of the mothers doing KMC. For example one participant in this study said:

"Everything was okay except that it was difficult the first time to kangaroo the child; I had the back sore ... backache ... you see" (Gloria).

Some participants temporarily discontinued 24-hour KMC in order to relieve their backs through slight exercises:

"I happen to get pain may be on my shoulder, my back; it is discomforting me; I stop the kangaroo; they [Nurses] just asked us why are you not doing $\mathrm{KC}$ and then you tell the reason; do some exercise may be for fifteen minutes or so" (Lissi);

"When you are tired you can let your baby on the crib, you are resting your back and you are resting yourself and then you take back your baby and kangaroo again" (Pearl).

Upon reflecting on their individual experiences of fatigue, all the participants in this study conceded not to use 24-hour $\mathrm{KMC}$ once discharged. Their decision was made mainly upon anticipation of other responsibilities that are expected of mothers in the home environment. For example, two participants said:

"I don't want to lie to you; I want to tell you straight; at home there are many things that you need to do; it is not like here; I am not going to do for 24 hours but will do most of the time" (Precious).

"It is going to be difficult because at home I will be doing some work; it is not like here where they cook for us, they do everything for us and only leave us to do kangaroo care and bathe ourselves" (Maggie).

\section{(iii). Sub-Theme3) It Is Very Hot}

All participants experienced high temperatures during $\mathrm{KMC}$ and felt these were responsible for their continuous exhaustion. Participants found it difficult to adjust to the high temperature caused by both the ward's temperatures and their bodies' heat in relation to direct skin contact with their preterm babies. The heaters were at the time of study regulated to the WHO's suggested environmental temperatures of 22-24 o C. (WHO, 2003:15). Two participants said:

"They opened the heaters; it is hot; the heaters are always open here; it is hot; we open the windows sometimes but sometimes are closed; they say the babies need to get warmth; it makes me feel so tired" (Rebecca).

"It is tiring; it is hot when the baby is here, skin to skin, though the heaters also contribute; you can't do anything out there when the baby is here; this is what I have experienced; you feel sleepy" (Maria).

The continued use of heaters was encouraged to maintain the recommended ward temperature as explained previously, especially given that the data was collected during the winter season where exterior temperatures ranged between 0 and 16 degrees centigrade. Moreover, mothers' feelings of exhaustion and sleepiness could be attributed to the heat generated through skin contact with their babies.

\section{Discussion}

This study sought to convey the experiences and perceptions of mothers towards 24-hour KMC at an academic hospital in Johannesburg, with findings showing that KMC initially seemed strange and difficult for all the participants during the first few days in the KMC ward but their satisfaction evolved gradually as they experienced its benefits. Amongst benefits reported are mothers' closeness as well as continual interactions with their babies, which then facilitated a maternal-infant attachment. Maternal-infant attachment a recurrent experience amongst all the participants has been described as a process emerging from maternal-infant interactions, maternal-infant closeness and maternal commitment to love and care for their babies during the KMC motherhood experiences [9]. These interactions involved gazing at each other, smiling, touching and responding to the baby's cry and breastfeeding. These findings are consistent with other past studies $[14,15]$ where KMC primarily promoted the attachment process between the mother and her newborn baby. Further, as attachment evolved, other benefits associated with KMC emerged, such as the baby's security and nurturing care. This may have occurred because attachment plays a role in promoting maternal nurturing 
behaviors that support a baby's growth and development [16]. Maternal-infant attachment during KMC also empowers the mothers' feelings, making them more responsible and confident in their capacity to care for their babies [9, 15].

The mother's physical and psychological discomfort resulting from exhaustion, a boring environment or the longing to go home due to separation from the rest of the family members and friends were among factors strongly involved in interfering with maternal-infant attachment during the 24-hour KMC practices at the academic hospital. Studies [17], note that in such state of mind, a mother may not be in a position to respond quickly to the baby's cry or provide gentle soothing measures that include smiling, playing or rocking. The attachment process may also not occur due to overt or covert processes of poor health status of the baby or the mother, environmental circumstances, and/or the quality of care that the baby receives from nurses and other care givers.

The current study and similar studies [7, 18, 19] further affirm KMC as an ideal hospital environment, which equips mothers with the knowledge and skills associated with motherhood responsibilities. The love and an enabling environment created by closeness between mother and child during $\mathrm{KMC}$ enhance a conducive environment for the learning of new skills and increases the mother's understanding of her baby's cues.

Nurse-mothers interactions in the current study improved mothers' satisfaction during the hospitalization of their babies. Trust created within the nurse-mother interaction is often the cornerstone for any quality care in many health care studies [18-20]. This may be due to the guidance, support and health care information received directly or indirectly from nurses. In our current study, direct information to the mothers was through health training provided by the nurses. Indirect information on the other hand, was through sharing of information in peer groups' forums of discussion created. The knowledge that the mothers acquired from the nurses and their own peer group is crucial for the care of their preterm babies as it provided empowerment, confidence and feelings of satisfaction at being able to do something positive for their preterm babies. When nurses are friendly, the mothers' fears of disease, strangeness and alienation are swapped with feelings of satisfaction, belonging and involvement and this clearly contributes to a deeper enjoyable experience.

Partnership in care enables mothers to shift from being passive observers to active participants. This occurs when nurses provide different kinds of information at different stages of their babies' hospitalization. When mothers are consistently informed about their babies' condition, they become knowledgeable and more likely to be active partners in caring for their babies, particularly in the areas of babies' health, babies' care, coping with and adapting to her individual baby [20].

Meeting other mothers with similar problems was perceived as one way of receiving support and satisfaction during this period of preterm birth and KMC experience. The support group during this period is helpful for providing mothers with care information and it connects them to a group of other mothers with similar problems [18]. Because of this connection, one mother in this study developed a strong commitment to care, realizing that she was not alone and that there were other similar babies in the KMC ward gaining weight and being discharged. Observing their babies' progress was a time of relief and joy, especially for those mothers who at the beginning felt that KMC was a waste of time.

Mothers take a sense of pride and satisfaction by being their babies' companion, observing their growth and providing maternal nurturing care. In addition to maternal nurturing care, babies' developmental progress in this study is attributed to a warm comfortable KMC environment resulting in the baby's ability to sleep quietly for longer periods of time and rapid weight gain. Weight gain is partly associated to the baby's ability to sleep longer, thus conserving energy and preserving caloric expenditure toward growth [17, 21]. With KMC, babies have been observed to spend longer hours sleeping, with other studies linking KMC to a baby's natural habitat [22]. This compares to the description of one participant in this study who remarked a similarity between the mother's uterus and $\mathrm{KMC}$, referring to it as a comfortable environment in which the baby can play and move around.

All participants nevertheless, encountered fatigue as their main challenge during the 24-hour KMC. This was associated with factors experienced in the KMC ward such as inadequate sleep, the use of one position for sleep and rest (supine/back position) leading to backache, high temperatures from skin-to-skin contact and the ward environment. Inadequate sleep was the result of interruptions to normal sleep cycles caused by the three-hourly feeding program of their preterm babies. This interruption of sleep therefore contributed to mothers' fatigue while providing 24-hour KMC. It is clear from the study that one complete sleep was not possible with the care of the pre-term newborns because each baby required three-hourly feeding. This made adjustment, both to KMC and to the environment, somewhat difficult for most of the mothers in the first few days. When the sleep pattern is not consistent, a desynchronization, or mismatching of the circadian biological rhythms occurs, thereby disrupting the timing of physiological and behavioral activity, which in turn causes fatigue. Further, mother's fatigue during the postnatal period as a consequence of the physical demands of caring for a newborn baby and that is augmented by disturbed sleep [1].

Participants found it difficult to adjust to the high temperatures caused both by the ward space heaters' temperatures and by their bodies' heat in relation to direct skin contact with their preterm babies. Literature [6] suggests that skin-to-skin contact during KMC enables a mother's breasts to generate heat in response to the thermal needs of the baby.

\section{Conclusion}

The 24-hour KMC positively influenced mothers' satisfactions and maternal-infant attachments during care. The nurse-mother interaction through health education, counseling and actual care of babies helped mothers in the healing process from their preterm birth experience and separation from their 
babies soon after birth. In addition, while fear of touch and separation initially hindered mothers from freely interacting with their preterm babies soon after birth, the introduction of 24-hour KMC alleviated fears of their babies' survival and enabled them to perceive their preterm babies in the same way as a normal baby. This in turn promoted a positive nurse-mother relationships and maternal-infant attachments. Nevertheless, while the 24-hour KMC is lauded for its advantages, mothers practicing it experience fatigue due to inadequate sleep occasioned by regular 3 hourly feeding schedules, high ward and skin to skin contact temperatures and one position of sleep and rest (supine position), which subsequently leads to backache and body exhaustion.

Considering KMC's benefits to the preterm baby, it should become institutionalized in all health care facilities on the continuum of maternal and new born care. Integration of KMC concept in the pre-service training curriculum of nurses will further sustain KMC practices in the care of preterm babies.

\section{Acknowledgements}

The authors acknowledge the contributions made by the CHB hospital management, Nurses and pediatricians in the 24-hr Kangaroo ward at the time of the study.

\section{Competing Interests}

The main author- Mr. Titus Kipchumba Tarus presented this paper for a Master of Science in nursing degree at the Witwatersrand University, under the supervision of the second author.

\section{References}

[1] Fraser D.M, \& Cooper M.A. Myles Textbook for Midwives. Philadelphia: Saunders 2003; 14th ed..

[2] World Health Organization [WHO]. Kangaroo mother care: a practical guide. Geneva: Department of Reproductive Health and Research, World Health Organization 2003.

[3] Whitelaw A. \& Sleath K. Myth of the Marsupial mother: home care of very low birth weight babies in Bogota, Colombia. Lancet, 1985; 1(8439): 1206-1208.

[4] Mokhachane M, Saloojee H, Cooper P. Earlier discharge of very low birth weight infants from an under-resourced African hospital: a randomized trial. Annals of Tropical Pediatrics, 2006; 26: 43-51.

[5] Charpak N, Ruiz-Pelaez J.G, Figueroa Z. Influence of feeding patterns and other factors on early somatic growth of healthy, preterm infants in home-based kangaroo mother care: a cohort study. Journal of Pediatric, Gastroenterology, and Nutrition, 2005; 41(4): 430-437.

[6] Ludington-Hoe S.M, Lewis T, Morgan K, Cong X, Anderson L, Reese, S. Breast and infant temperatures with twins during shared kangaroo care. Journal of Obstetric, Gynecologic, \& Neonatal Nursing, 2006; 35(2): 223-231.
[7] Roller, C.G. Getting to Know You: Mothers' Experiences of Kangaroo Care. Journal of Obstetric, Gynecologic, and Neonatal Nursing, 2005; 34(2): 210-217.

[8] Bergman N.J, Linley L.L, Fawcus S.R. Randomized controlled trial of skin to skin contact from birth versus conventional incubator for physiological stabilization in 1200-2199g newborns. ActaPaedriatr, 2004; 93(6): 779-785.

[9] Feldman R, Eidelman A.I, Sirota L, Weller A. Comparison of Skin-to-Skin (Kangaroo) and traditional care: parenting outcomes and preterm infant development. Pediatrics, 2002; 110(1): 16-26.

[10] Conde-Agudelo A, Beliza J.M, Diaz-Rosello J. L. Kangaroo mother care to reduce morbidity and mortality in low birth weight infants. Cochrane Database Syst Rev. 2011 Mar 16 ; (3):CD002771. doi: 10.1002/14651858.CD002771.pub2

[11] Kirsten G.F, Bergman N.J, Hann F.M. Kangaroo mother care in the nursery. Pediatric clinics of North America, 2001; 48(2):443-452.

[12] Ramanathan K, Paul V.K, Deorari A.K, Taneja U, George G. Kangaroo mother care in very low birth weight infants. Indian Journal pediatr, 2001; 68(11):1019-1023.

[13] Colaizzi, P. Psychological research as the phenomenologist views it. In: Valle, R.S. and King, M. (Eds), Existential phenomenological alternatives for psychology, New York: Oxford University Press, 1978.

[14] Klaus M.H. Mother and infant: Early emotional ties. Pediatrics, 1998; 102(5): 1244-1246.

[15] Tessier R, Cristo M, Velez S, Giron S.W, Calume Z.F, Juan G, et al. Kangaroo Mother Care and the Bonding Hypothesis. Pediatrics, 1998; 102(2): e17

[16] Dodd, V.L. Implications of Kangaroo care for growth and development in preterm infants. Journal of Obstetric, Gynecologic and Neonatal Nursing, 2005; 34(2): 218-232.

[17] Melnyk B.M, Feinstein N.F, Alpert-Gillis L, Fairbank S.E, Crean H.F, Sinkin R.A, et al. Reducing premature infants' length of stay and improving parents' mental health outcomes with the creating opportunities for parent empowerment (COPE) neonatal intensive care unit program: A randomized controlled trial. Pediatrics, 2006; 118(5): e1414-e1427.

[18] Mercer R.T. Nursing Support of the process of becoming a mother, Journal of Obstetric, Gynecologic, and Neonatal Nursing, 2006; 35(5): 649-651.

[19] Mercer R.T. \& Walker L.O. A review of nursing interventions to foster becoming a mother. Journal of Obstetric, Gynaecologic, and Neonatal Nursing, 2006; 35(5): 568-582.

[20] Mok E. \& Leung S.F. Nurses as providers of support for mothers of premature infants. Journal of clinical nursing, 2006; 15(6): 726-734.

[21] McCain G.C, Ludington-Hoe S.M, Swinth J.Y, Hadeed A.J. Heart rate variability responses of preterm infant to kangaroo care. Journal of Obstetric, Gynaecologic, and Neonatal Nursing, 2005; 34(6): 689-694.

[22] Ferber S.G, \& Makhoul I.R. The Effect of Skin-to-Skin Contact (Kangaroo Care) Shortly After Birth on the Neurobehavioral Responses of the Term Newborn: A Randomized, Controlled Trial. Pediatrics, 2004; 113(4): 858-865. 